\title{
Estudio de prevalencia de lesiones por presión en un Hospital Universitario, Bogotá-Colombia
}

\author{
Study of prevalence of pressure ulcers in a University Hospital, Bogota-Colombia \\ Estudo de prevalência de lesões por pressão em um Hospital Universitário, Bogotá- \\ Colômbia
}

\author{
Renata Virginia González-Consuegra* \\ Karen Tatiana Roa-Lizcano ** \\ Wilmar José López-Zuluaga ***
}

\section{Resumen}

Objetivo: Determinar la prevalencia de las LPP (Lesiones por presión) en pacientes hospitalizados, entre septiembre y noviembre del año 2016, en un Hospital Universitario de Bogotá, Colombia. Materiales y Métodos: Estudio descriptivo, transversal, de prevalencia de periodo que utilizó como instrumento una encuesta elaborada a partir de las directrices del Grupo Nacional para el Estudio y Asesoramiento de Úlceras por Presión y Heridas Crónicas GNEAUPP 2003. Los datos recolectados se obtuvieron a partir de las historias clínicas, valoración de enfermería y preguntas directas. Resultados: Se encontraron 40 pacientes con LPP en 282 camas, para una prevalencia de un $14.1 \%$; predomina el sexo masculino con un $67.5 \%$; las edades oscilan entre los 75 y 85 años. El $40 \%$ estaba hospitalizado en medicina interna, la mayoría pertenecía al régimen subsidiado en un $87.5 \%$. La etiología de la lesión fue de $92.5 \%$ por presión y de un $47.5 \%$ por humedad, clasificadas en fases 1 y 2 el $30 \%$. Se encontraron pacientes con más de dos LPP en un $30 \%$, la localización más común fue el talón con un $30 \%$. Se acentúa el desconocimiento sobre el uso de los Ácidos Grasos Hiperoxigenados en las superficies especiales para el manejo de la presión. Conclusión: Como resultado se observa que el estudio es similar a otros realizados en el país. Igualmente, se percibe que datos como las características de las LPP, su etiología y las medidas de prevención utilizados en la institución, son la base para el análisis general del fenómeno, ya que permite conocer el comportamiento de las LPP en el contexto nacional. Así mismo, se constituyen en insumos que contribuyen a construir un plan de cuidado integral así como su implementación, a partir de medidas educativas que sensibilicen al equipo de salud sobre la atención segura del paciente.

Palabras clave: Úlceras por presión (UPP), atención de enfermería.

\section{Abstract}

Objective: To determine the prevalence of pressure ulcers in hospitalized patients, between September and November of 2016, in a University Hospital of Bogota,

\section{Autor de correspondencia}

* $\triangle$ Enfermera. Magister en Epidemiología Prevención y tratamiento de heridas crónicas. Doctora en Salud Pública. Docente Universidad Nacional de Colombia. (iD https://orcid. org/0000-0001-9269-7789. Bogotá, Colombia.

** Enfermera. Magíster en Enfermería. Docente Universidad Nacional de Colombia. Correo:ktroal@unal.edu.co. (D) https://orcid. org/0000-0002-4390-9922. Bogotá, Colombia.

${ }^{* * *}$ Enfermero. Especialista en Epidemiología (C), Fundación Universitaria Juan N. Corpas. Universidad Nacional de Colombia. Correo: wjlopezz@unal.edu.co. (D) https://orcid. org/0000-0002-7260-2794. Bogotá, Colombia.

Este es un artículo bajo la licencia CC BY (https://creativecommons.org/ licenses/by/4.0/) @) (i) 
ISSN-PRINT

1794-9831

E-ISSN 2322-7028

Vol. 15 No. 2

Jul - Dic 2018

Cúcuta, Colombia

Colombia. Materials and Methods: Descriptive, cross-sectional study over a period of time, that used as an instrument a survey elaborated from the guidelines of the National Study and Assessment Group of Pressure Ulcers and Chronic Injuries GNEAUPP 2003. The collected data was obtained from medical records, nursing assessment and direct questions. Results: 40 patients with pressure ulcers were found in 282 beds, for a prevalence of $14.1 \%$; the male sex is predominant with $67.5 \%$; the ages range between 75 and 85 years old. $40 \%$ of the patients were hospitalized in internal medicine, the greatest proportion belonging to the subsidized regime $(87.5 \%)$. The etiology of the injury was of $92 \%$ for pressure and $47.5 \%$ for humidity, with $30 \%$ classified in phases 1 and $2.30 \%$ of the patients had more than two pressure ulcers, the most common location was the heel (30\%). It is emphasized that there is a lack of knowledge about the use of hyper oxygenated fatty acids in special surfaces for the treatment of the pressure. Conclusion: it is observed that the study is similar to other studies performed in the country. Also, it is perceived that data such as the characteristics of pressure ulcers, its etiology and the measures of prevention used in the institution, are the base for the general analysis of the phenomenon, since it allows to understand the behavior of the pressure ulcers in a national context. Similarly, these become a key factor that contribute to build a plan of comprehensive care as well as its implementation through educative measures that sensitize the health team about the safe care of the patient.

Keywords: Pressure ulcers, nursing care.

\section{Resumo}

Objetivo: Determinar a prevalência das LPP (Lesões por pressão) em pacientes hospitalizados, entre setembro e novembro do ano 2016, num Hospital Universitário da cidade de Bogotá, Colômbia. Materiais e Métodos: Estudo descritivo, transversal, de prevalência de período que utilizou como instrumento uma pesquisa elaborada a partir das diretrizes do Grupo Nacional para o Estudo e Assessoramento de Úlceras por Pressão e Feridas Crónicas GNEAUPP 2003. Os dados coletados se obtiveram a partir das histórias clínicas, valoração de enfermagem e perguntas diretas. Resultados: Se encontraram 40 pacientes com LPP em 282 camas, para una prevalência de um 14,1 $\%$; predomina o sexo masculino com um $67,5 \%$; as idades oscilam entre os 75 e 85 anos. O 40 $\%$ estava hospitalizado em medicina interna, a maioria pertencia ao regímen subsidiado num 87,5 $\%$. A etiologia da Lesão foi de $94,0 \%$ por pressão e de um $47,5 \%$ por umidade, classificadas em fases 1 e 2 ou $30 \%$. Se encontraram pacientes com mais de dois LPP num $30 \%$, a localização mais comum foi o calcanhar com um $30 \%$. Se acentua o desconhecimento sobre o uso dos Ácidos Graxos Hiperoxigenados nas superfícies especiais para o manejo da pressão. Conclusão: Como resultado se observa que o estudo é similar a outros realizados no país. Igualmente, se percebe que dados como as características das LPP, sua etiologia e as medidas de prevenção utilizadas na instituição, são a base para a análise geral do fenómeno, já que permite conhecer o comportamento das LPP no contexto nacional. Assim mesmo, se constituem em insumos que contribuem a construir um plano de cuidado integral assim como sua implementação, a partir de medidas educativas que sensibilizem a equipe de saúde sobre $\mathrm{o}$ atendimento segura do paciente.

Palavras-chave: Úlceras por pressão (UPP), atendimento de enfermagem.

\section{Introducción}

Las lesiones por presión (LPP) son consideradas heridas crónicas que se han encontrado presentes en la humanidad desde que existen la enfermedad, la discapacidad, la inmovilidad y la ancianidad debilitante, lo que explica porqué este no es un fenómeno nuevo, lo que ratifica la importancia de su estudio (1).
Las LPP han sido ampliamente definidas según diversos autores. Soldevilla en el estudio Epidemiología, coste y repercusiones legales de las úlceras por presión en España, años 2005-2006 las define como "lesión localizada en la piel o el tejido subyacente, por lo general sobre una prominencia ósea, como resultado de la presión (incluyendo presión en combinación con cizalla)" (2). La guía de práctica clínica de enfermería: prevención y tratamiento de las LPP y heridas crónicas 
amplían dicho concepto al señalar que son lesiones de origen isquémico ocasionadas por presión, fricción y cizalla sobre un plano o prominencia ósea, bloqueando el flujo sanguíneo en el tejido subyacente, produciendo isquemia y rápida degeneración de los tejidos (3). También se las definió como "Una lesión localizada en la piel y/o en el tejido subyacente en general sobre una prominencia ósea, como resultado de la presión, o la presión en combinación con las fuerzas de cizallas. En ocasiones, puede aparecer sobre tejidos blandos sometidos a presión externa por diferentes materiales o dispositivos clínicos. Sin embargo, recientemente se dio un cambio de terminología y se cambió de "Úlceras por presión" a "lesiones por presión" definidas como el daño a la piel y / o tejido blando subyacente generalmente sobre una prominencia ósea o relacionada con un dispositivo médico o de otro tipo. La lesión puede presentarse en la piel intacta o como una úlcera abierta y puede ser dolorosa, se produce como resultado de la presión o presión intensa o prolongada en combinación con cizallamiento. La tolerancia de los tejidos blandos para la presión y el cizallamiento también puede verse afectada por el microclima, nutrición, perfusión, comorbilidades y condición de los tejidos blandos (4).

Así mismo las LPP se clasifican en fases según The National Pressure Ulcer Advisory Panel (NPUAP) (5), quienes las catalogan en cuatro fases y tres especiales:

Fase 1: Denominado como eritema no blanqueable de la piel intacta. Puede aparecer en piel oscuramente pigmentada y manifestarse con eritema que no sede o con cambios en la sensación térmica. Incluye decoloración púrpura o marrón, puede indicar lesiones profundas (5).

Fase 2: Pérdida parcial de la piel con dermis expuesta. El lecho de la herida es viable, de color rosa o rojo, húmedo o estar intacto. Puede presentar ampolla intacta o no. No es visible el tejido adiposo. Las lesiones suelen resultar de microclimas adversos (5).

Fase 3: Pérdida total de la piel y de su espesor, es visible el tejido adiposo, el de granulación y el epibolio (bordes enrollados de la herida). Hay presencia de esfacelos, lesiones a profundidad del tejido y presencia de socavaciones y túneles. No hay exposición de fascia, músculo, tendón, ligamento, cartílago o hueso (5).
Fase 4: Pérdida total del espesor de la piel y exposición del tejido: fascia, músculo, tendón, ligamento, cartílago o hueso. Hay presencia de epíbole y en ocasiones túneles. La profundidad depende de la ubicación anatómica (5).

Lesión por presión no estadiable: Pérdida total de la piel, del espesor y de la extensión del tejido. El daño es difícil de establecer (5).

Lesión profunda del tejido: Piel intacta o no, con área localizada blanquecina, de color rojo oscuro, marrón o púrpura. El lecho de la herida puede ser oscuro con ampolla llena de sangre. La lesión suele resultar de una presión intensa y / o prolongada. La herida puede evolucionar rápidamente para revelar la extensión real de la lesión tisular (5).

Lesiones de mucosa: Corresponde a lesiones por presión en las membranas mucosas (5).

La etiología de las LPP consignada en la literatura científica desde los años ochenta, ha demostrado la influencia que tienen las fuerzas mecánicas y la humedad como factores desencadenantes en la aparición de las LPP, reafirmando su carácter multifactorial. Diversos autores refieren que dentro de las principales causas de su manifestación están: la presión, la cual puede distinguirse en tres tipos de mecanismos y la presión directa como fuerza ejercida de forma perpendicular entre la piel y las prominencias óseas; la fricción como una fuerza ejercida de manera paralela al paciente sobre un plano duro o semiduro, causada por el arrastre del paciente o durante su movilización; y el cizallamiento como una fuerza sumatoria entre la fuerza tangencial y la presión directa que ocasiona daño a los tejidos internos del paciente (3). Adicionalmente la humedad es considerada como un factor causal de LPP (6).

Así mismo, se ha confirmado que diversos factores de riesgo tanto intrínsecos como extrínsecos pueden influir en su aparición como: los trastornos neurológicos, las alteraciones nutricionales, el tratamiento con inmunosupresores y con sedantes, los trastornos en la alteración del oxígeno, la espasticidad y las contracturas musculares. También se ha comprobado que este tipo de lesión puede presentarse en menores de 36 meses con bajo peso al nacer, así como en mayores de 70 años (3).
E-ISSN 2322-7028

Vol. 15 No. 2

Jul - Dic 2018

Cúcuta, Colombia 
Cabe considerar por otra parte, que uno de los efectos más relevantes que traen consigo las LPP es el problema de salud pública que afecta a todos los niveles asistenciales como ha sido demostrado en múltiples publicaciones científicas del mundo (7-12), específicamente en España $(1,13,14)$. Dicha problemática afecta a millones de personas en el mundo y trae consigo múltiples complicaciones como : dolor, infección local y sepsis, alterando aún más el estado de salud, comorbilidades y deterioro de la calidad de vida, situaciones que pueden llevar al paciente a la discapacidad y a la muerte $(12,14)$.

Adicionalmente, las LPP alargan la estancia hospitalaria, aumentan el costo de los recursos necesarios para la atención de este tipo de pacientes, así como la carga laboral del equipo de enfermería $(12,15)$. Lo anterior es perfectamente evitable hasta en un $98 \%$ si se implementan medidas de prevención, enmarcadas en políticas institucionales para la seguridad del paciente, orientadas hacia la instauración de cuidados de la piel y normas preventivas que sirvan para disminuir la aparición de LPP en la población de riesgo (7).

Debe señalarse que desde el año 2002 en Colombia, el Ministerio de la Protección Social, crea el Sistema Obligatorio de Garantía de Calidad de la Atención en Salud que ha impulsado la política de la Seguridad del Paciente, concretado con la promulgación de la resolución 1446 de 2006, en donde se consideran las LPP como un evento adverso a tener presente en la atención sobre salud (16).

Por último, es conviente anotar que en Colombia son escasos los estudios y datos relacionados con la incidencia y prevalencia de LPP, lo cual dificulta realizar un análisis situacional del país. Sin embargo, un estudio de prevalencia realizado en 2015 encontró una prevalencia de LPP de 2.2 (12). No obstante, en distintos lugares del mundo, particularmente en Europa, existe una amplia y rigurosa bibliografía sobre este tema, sin desconocer que en latinoamerica también se han adelantado algunos estudios al respecto $(2,12,17-22)$.

En la perspectiva que aquí se adopta, con el presente estudio se quiere contribuír desde lo social, a dar visibilidad a un grupo pobacional que es víctima de LPP; además, se coadyuva en la construcción de rutas de investigación, brindando herramientas orientadoras a las enfermeras. Del mismo modo, se puede identificar a la población con LPP, lo que permitirá dar el primer paso en la formulación de planes de acción tendientes a mejorar la calidad de vida de los pacientes

\section{Objetivo}

- Determinar la prevalencia de las LPP (Lesiones por presión) en pacientes hospitalizados entre septiembre y noviembre del año 2016, en un Hospital Universitario de Bogotá, Colombia.

\section{Materiales y Método}

Esta investigación se realizó en una institución de salud universitaria de tercer nivel en la ciudad de Bogotá, por medio de un estudio cuantitativo de tipo descriptivo y de corte transversal. Se tuvo como objetivo determinar la prevalencia de las LPP, durante los meses de septiembre a noviembre del 2016 en los todos los servicios del hospital, a excepción de Ginecobstetricia y Neonatología por no contar con avales para estas áreas. Se tomó como numerador la cantidad de pacientes con LPP, siendo un total de 40 y como denominador las 282 camas con que cuenta la institución.

En la primera etapa se elaboró un cuestionario basado en los lineamientos de la GNEAUPP en el cuarto estudio nacional de prevalencia (2) donde se consignó la información recolectada.

En la segunda etapa se diligenció el instrumento utilizando como fuente de información a los enfermeros de los diferentes servicios; se complementó con los registros de las historias clínicas de los pacientes teniendo en cuenta: aspectos demográficos, tiempo de evolución, etiología de las lesiones y lugar de procedencia. De igual forma, se valoraron las lesiones que tienen que ver con: localización, fases y número de lesiones y la anamnesis en cada uno de los pacientes con el fin de complementar información de la historia clínica; finalmente, se consignaron las respuestas de enfermería sobre el uso de escalas de valoración, cambios de posición y el uso de productos para el cuidado de la piel durante la atención.

En la tercera etapa se consolidó la información en Excel y posterior a su depuración se realizó el análisis mediante estadísticas descriptivas en el aplicativo Epi info 7.0. 
El estudio se ha clasificado sin riesgo, ya que los participantes accedieron a ser valorados de forma voluntaria, se les garantizó la confidencialidad de los datos y su utilización con propósitos académicos y científicos; además, se contó con el aval del comité de ética de la institución de donde se obtuvo la información.

\section{Resultados}

La prevalencia total hallada fue $14,1 \%$, con predominio en los hombres con un $67.57 \%$. Las LPP se presentaron con mayor frecuencia en pacientes cuyas edades oscilan entre 75 y 85 años con un 27.5 $\%$, seguidos del $25 \%$ que tiene entre 65 a 75 años, luego está el $17.5 \%$ conformado por quienes tienen entre 85 y 95 años y finalmente está el grupo de 55 a 65 años con $15 \%$ (Ver Grafica 1). Las LPP la padecen con más frecuencia las personas con piel de color blanco en un $67.5 \%$, le siguen las de piel trigueña en un $30 \%$ y con un $2.5 \%$ las de piel negra.

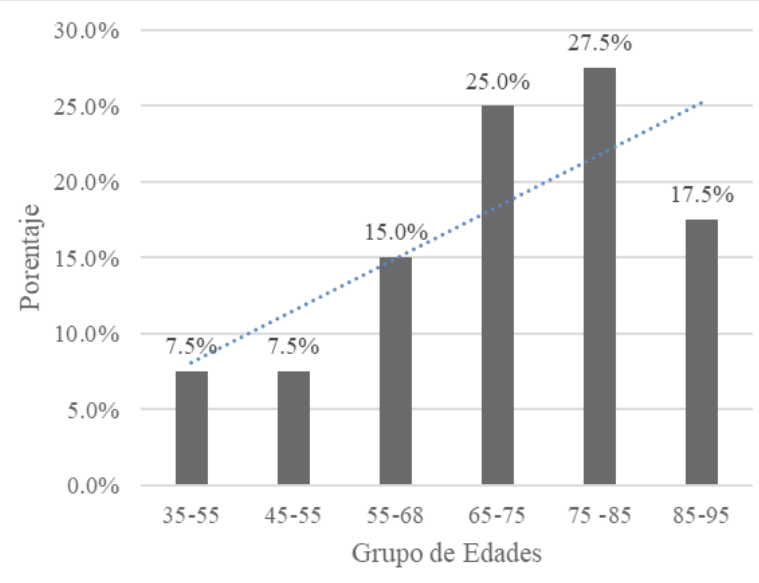

Grafica 1. Frecuencia de las LPP por grupo de edades en un hospital universitario de Bogotá

Fuente: Elaboración propia

El servicio en el que prevalecen los casos de LPP fue el de Medicina interna con un $40 \%$, seguido por Ortopedia con $22,5 \%$ y la unidad de cuidados intensivos UCI con un 12,5\%; los servicios de Urgencias y Cirugía presentaron un $10 \%$, cada uno y Neurocirugía el $5 \%$. Por otra parte, el 87,5\% de los pacientes con LPP pertenecían al régimen subsidiado (Ver Grafica 2).

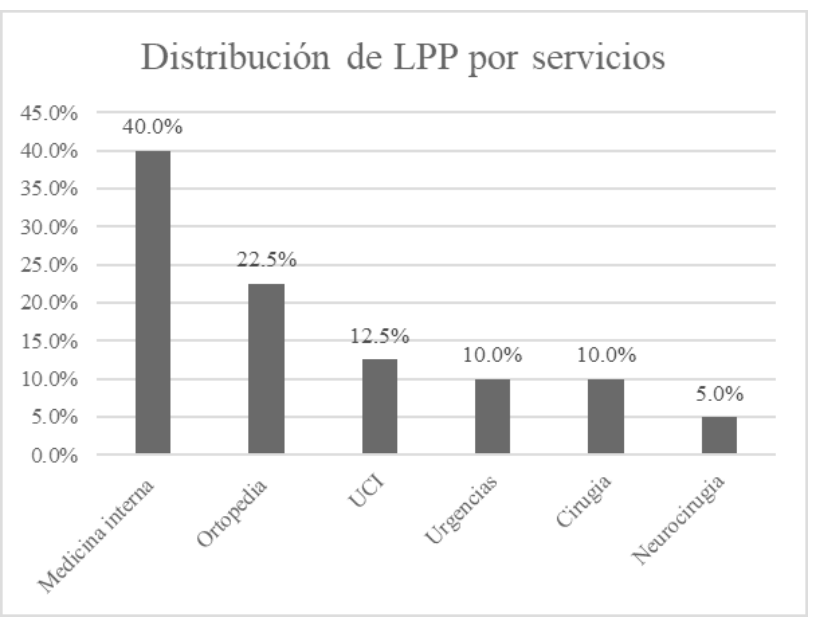

E-ISSN 2322-7028

Vol. 15 No. 2

Jul - Dic 2018

Cúcuta, Colombia

Grafica 2. Frecuencia de LPP por servicios en un hospital universitario de Bogotá

\section{Fuente: Elaboración propia}

La localización de LPP en los talones es la más frecuente con un $30 \%$, seguido de la región sacra $17.5 \%$, los maléolos y trocánteres $12.5 \%$ cada uno, los codos con un $10 \%$, hombros y mano $5 \%$ en cada caso, glúteos y rodillas $2.5 \%$ respectivamente (Ver grafica 3).

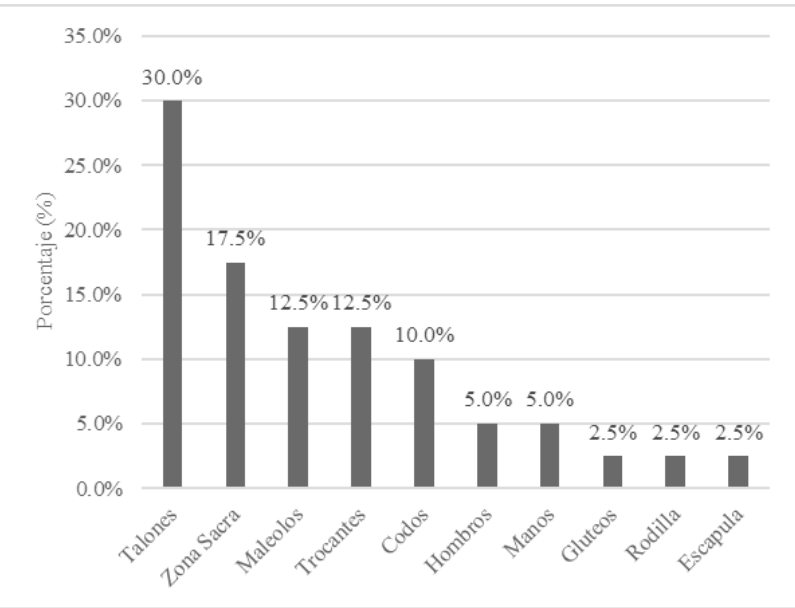

Gráfica 3. Frecuencia de LPP según la ubicación anatómica en un hospital universitario de Bogotá.

Fuente: Elaboración propia

Un solo paciente puede tener varias LPP en diferentes fases. Para este análisis se agruparon de acuerdo al marco de referencia (1), teniendo en cuenta las fases: se encontró que en la I y II combinadas son las de mayor frecuencia con un $30 \%$; seguido de la I 
ISSN-PRINT

1794-9831

E-ISSN 2322-7028

Vol. 15 No. 2

Jul - Dic 2018

Cúcuta, Colombia y la II con un $25 \%$ cada una; en la fase IV con un 7.5 $\%$;un $5 \%$ en las II y III; en la I y III $2.5 \%$ y las I, II y III con un $2.5 \%$. La fase indeterminada ocupó el $2.5 \%$. Se explica el porcentaje de ésta última, por la dificultad al casificarla o por encontrarse cubierta al momento de la valoración, y se deseaba no alterar el tratamiento implementado (Ver grafica 4).

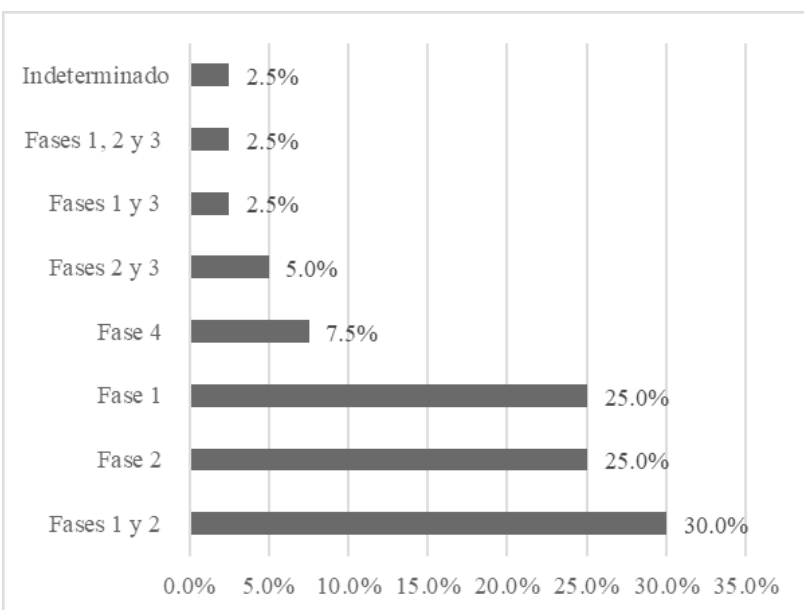

Grafica 4. Frecuencia de LPP según su fase, en un hospital universitario de Bogotá

Fuente: Elaboración propia

En cuanto a la antigüedad de las LPP, se clasificaron en 4 grupos: de 0 a 2 meses, de 2 a 4 , de más de 24 meses y de tiempo indeterminado, por no contarse con registros en la historia clínica, ni información suministrada por el paciente o familiar. De acuerdo al tiempo de evolución se encontraron los siguientes porcentajes: el $72.5 \%$ de las LPP evolucionan entre 0 a 2 meses, el $15 \%$ en un tiempo de evolución indeterminado, el $10 \%$ entre 2 a 4 meses y el $2.5 \%$ alcanzan un tiempo mayor a 24 meses.

La etiología de las LPP se relaciona principalmente con la presión ejercida en los tejidos en el $92.5 \%$. Sin embargo, es necesario considerar la multicausalidad en la etiología de las LPP, así: el $30 \%$ de los casos son la combinación de presión con cizalla, el 22.5 $\%$ conjunción entre la presión con humedad e incontinencia, el $15 \%$ es presión con humedad así como presión con incontinencia, el $10 \%$ es presión con humedad, incontinencia y cizalla, y el $7.5 \%$ no fue posible determinar su etiología.

Los hallazgos muestran que un paciente puede presentar varias LPP, así: el $35 \%$ tenía una LPP, el $35 \%$ dos simultáneamente, $20 \%$ presentaron tres a la vez y $10 \%$ de los casos tenían cuatro LPP al mismo tiempo. Según el lugar donde se origina la LPP están: la hospitalización con un $72.5 \%$, seguido de las LPP provocadas en casa con un $20 \%$ y por último las que se producen en el lugar de donde fue remitido el paciente con un $7.5 \%$.

Considerando las medidas de prevención, en cuanto al uso de Escalas de Valoración del Riesgo de LPP (EVRUPP), el $100 \%$ de los profesionales de enfermería manifiesta utilizar la escala de NOVA establecida por protocolo institucional; sin embargo, se desconoce su validación en Colombia.

E1 $100 \%$ de los enfermeros respondieron que realizan cambios de posición a sus pacientes como medida de prevención de LPP. A este respecto, el $95 \%$ indicaron que realizan los cambios de posición cada 120 minutos, mientras que el $5 \%$ señalaron que los realizan cada 240 minutos.

En cuanto a los productos utilizados para el cuidado de la piel, el $90 \%$ de los enfermeros manifestaron que utilizan crema hidratante, el $5 \%$ emplean cremas hidratantes y película transparente, el $3 \%$ no usa ningún producto y el $2 \%$ aplica crema hidratante y crema de Marly.

La totalidad de los enfermeros refirió que no disponen de superficies especiales para el manejo de la presión (SEMP). El $95 \%$ de los profesionales de enfermería exponen que no conocen los ácidos grasos hiperoxigenados

(AGHO) como parte de las estrategias de prevención de lesiones de piel, el $5 \%$ no los utiliza. El 92 $\%$ manifiesta desconocer demandas jurídicoLegales relacionadas con LPP.

\section{Discusión}

La prevalencia de LPP en la institución fue del 14,1 $\%$, cifra inferior respecto a estudios similares realizados en Bogotá y Cartagena, los cuales reportaron una prevalencia de $18 \%$ y $24 \%$ respectivamente $(23,24)$. No obstante, según hallazgos encontrados en Bucaramanga muestran una prevalencia de 5,27 $\%$ (25). Pese a todo, el último estudio nacional de prevalencia es congruente con los resultados del presente estudio de 5,2 (26).

La mayor prevalencia se encuentra en medicina interna con un $40 \%$, similar a los resultados de Cartagena que tiene un $38 \%$ en el mismo servicio, 
seguido de ortopedia con un $22 \%$ (23); resultados que difieren del estudio realizado en Bogotá cuya mayor prevalencia fue en UCI con un $31.25 \%$, seguido de medicina interna con un $17.64 \%$ (24). Cifras que coinciden con la investigación de González quien señala que en el mismo servicio hay un $41 \%$, seguido de ortopedia con un $7 \%$ (12); porcentajes atribuibles a múltiples factores de riesgo relacionados con el estado de salud y la edad; factores que se visibilizan en este estudio debido a que los grupos con mayor prevalencia son los pacientes que tienen entre 75 y 85 años, seguidos del grupo entre 65 y 74 años quienes generalmente se encuentran en el servicio de medicina interna debido a que sufren enfermedades crónicas, con sus respectivas complicaciones, lo que aumenta el riesgo de tener LPP y por tanto demanda mayores cuidados de enfermería.

Los resultados muestran que las LPP en esta institución se presentan desde la edad de 35 años predominando en personas de la tercera edad, los cuales concuerdan con reportes de la literatura en diferentes lugares del mundo como en México con $58.3 \%$ (27) y Brasil con un $66.7 \%$ (7).

Del mismo modo, los resultados sobre la incidencia de los LPP revelan que un $67.5 \%$ prevalece en hombres, similar a la información suministrada por Arjona quien indica que en un hospital de tercer nivel en Cartagena son el 61\% (23); así como Valero et al., quienes señalan que en Bucaramanga hay un $59.4 \%$ de pacientes hombres (25). También, coinciden los hallazgos de Bautista y Bocanegra al indicar que en un hospital universitario de tercer nivel de la ciudad de Bogotá el $55.7 \%$ son hombres (24). A nivel mundial, países como China no es la excepción, pues según Zhao el $56.6 \%$ de los pacientes son de sexo masculino (10).

De igual manera, el color de piel más afectado por LPP es la blanca con el $67.5 \%$, seguido de los pacientes con piel trigueña con un $30 \%$ y por último los de piel negra con el $2.5 \%$. Sin embargo, en este estudio no se puede precisar sobre la incidencia de Úlceras por presión en un color de piel en particular, ya que no se conoce el censo total de pacientes de la Institución sobre los pacientes clasificados por raza, factor que permitirá realizar investigaciones a futuro sobre este tema. Sin embargo, se han encontrado algunos estudios en los que se demuestra que existe una mayor incidencia de LPP en personas de raza blanca (28); aunque, algunos autores han asegurado que no existen suficientes estudios en los que se indique si los LPP afectan a la población latina, que permitan evaluar esta condición (29).

De igual manera, se puede indicar que la región corporal con mayor frecuencia de LPP son los talones con un $30 \%$, seguida de la zona sacra con el $19 \%$, los tobillos y los trocánteres con un $12 \%$ respectivamente; estos resultados coinciden con un estudio realizado por la Universidad Javeriana donde el talón ocupó el primer puesto con una prevalencia de $33,3 \%$ y la zona sacra con un $28.5 \%$ (24). Sin embargo, un estudio de prevalencia realizado en la Universidad Nacional de Colombia muestra que la zona sacra con un $24 \%$ y el trocánter con el $19 \%$ (12) son las zonas más afectadas, lo cual coincide con otro estudio realizado en Cartagena donde la zona sacra ocupó el primer lugar con un $46 \%$ seguida del talón con el $15 \%(23)$.

Según este estudio, la fase más afectada por las LPP corresponde a las I y II con un $30 \%$, seguidas del estadío II con un $25 \%$, al igual que el estadío I; el IV alcanza el 7,5\%; simultáneamente las fases II y III representan el $5 \%$. Así mismo, el 2,5\% corresponde al I y III respectivamente; finalmente las fases I, II y III tienen un 2,5\%. La fase indeterminada obtuvo un 2,5\%, debido a que estaba cubierta al momento de la valoración, y se deseaba no afectar el tratamiento implementado. Estudios similares en Estados Unidos muestran el predominio del estadio II (11), al igual que en España, seguidos del III, I y IV (14). Por su parte México clasifica las LPP en los estadios I y II sin progresión a estadios más avanzados (27); en Brasil las LPP representan la mayoría en el II, sin embargo ocupan un lugar importante en el I (7). En un estudio realizado en Chile los LPP se manifiestan en los estadíos I, II y III (20). De otra parte, en Colombia según estudios ya referenciados, en Bogotá y Cartagena se evidencia el predominio de LPP en los estadíos I y II $(12,23)$ y en Bucaramanga lo más frecuente está en el estadío III (25). Es de resaltar, que en la presente investigación se hace un análisis sobre la aparición simultánea de LPP en los pacientes, el cual permite ver la complejidad del problema en conjunto.

Respecto a la etiología de la LPP la presión interviene en un $92.5 \%$ de los casos distribuídos como sigue: presión y cizalla el $30 \%$, presión con humedad el 
ISSN-PRINT

1794-9831

E-ISSN 2322-7028

Vol. 15 No. 2

Jul - Dic 2018

Cúcuta, Colombia
$15 \%$, presión con humedad e incontinencia el 22.5

$\%$, presión con incontinencia el $15 \%$, presión con humedad y cizalla el $10 \%$. Llama la atención que los estudios revisados no examinan las variables relacionadas con la presión, cizalla y humedad; únicamente tienen en cuenta la incontinencia tanto la urinaria como la fecal. Según las conclusiones del Grupo Español de Expertos de GNEAUPP hay siete lesiones de piel, producidas con relación a la dependencia (30).

En cuanto a la EVRUPP, se encontró que el $100 \%$ de los enfermeros utilizan NOVA como protocolo institucional al ingreso de los pacientes, en contraste con otros estudios que reportan que las EVRUPP más utilizadas son las escalas de Norton modificada, Norton (31) y Braden, siendo ésta última validada en Colombia (32), por tanto es la que se recomienda utilizar $(7,9,25)$.

Respecto a los métodos de prevención de LPP, si bien los enfermeros y auxiliares de enfermería en su mayoría realizan cambios de posición de sus pacientes cada dos horas; sin embargo, manifiestan que la alta carga laboral y la falta de insumos impide realizar cambios de posición con la frecuencia deseada, así como utilizar la implementación de otros cuidados de la piel tales como la hidratación, limitaciones que atentan contra la seguridad del paciente.

\section{Conclusiones}

- Como resultado de este estudio se obtuvo una prevalencia similar a otros realizados en el país, que aunados a los demás datos obtenidos como características de las LPP, etiología y medidas de prevención utilizados en la institución, servirán de base para el análisis general del fenómeno, lo que permite conocer el comportamiento de las LPP; así mismo, se constituyen en insumos que contribuyan a construir un plan de cuidado integral y a su implementación, a partir de medidas educativas que sensibilicen al equipo de salud sobre la atención segura del paciente.

- También se propone que a futuro se viabilicen políticas institucionales que promuevan la cualificación de los equipos de salud hacia la calidad de la atención y los servicios allí prestados.

- Se necesita un trabajo conjunto interdisciplinario para diseñar y aplicar estrategias de prevención de LPP de manera constante y prolongada en el tiempo, que sean garantes del impacto significativo institucional. De igual manera, es necesario el respaldo presupuestal institucional con el fin de invertir en recursos reales, evaluando su costo y efectividad en relación con la prevención y el tratamiento de las LPP.

- Siguiendo las políticas institucionales de formación, capacitación e investigación, es importante realizar un seguimiento continuo que privilegie el uso de estrategias de prevención tales como: guías de práctica clínica fundamentadas en la evidencia científica y uso de escalas de valoración apropiadas.

\section{Conflicto de intereses}

Los autores manifiestan no tener conflicto de intereses.

\section{Referencias Bibliograficas}

1. Gonzalez Consuegra RV, Matiz GD, Guzman XL, Hernández JD, Mora LH, Celis JS. Intervención y cuidado de enfermería a personas con lesiones de piel. Úlceras por presión y dermatitis asociada a incontinencia urinaria, fecal o mixta. Bogotá D.C.: Universidad Nacional de Colombia; 2016.

2. Pancorbo Hidalgo PL, García Fernández FP, Torra I Bou JE, Verdú Soriano J, Soldevilla Agreda JJ. Epidemiología de las úlceras por presión en España en 2013: 4.o Estudio Nacional de Prevalencia. Gerokomos. 2014; 25(4):162-170.

3. Martínez Aviléz MJ, Sánchez Llorente MM. Guía de Práctica Clínica para el cuidado de personas con úlceras por presión o riesgo de padecerlas. Consejeria de Sanitat.eneralitat Valenciana; 2012.

4. The National Pressure Ulcer Advisory Panel (NPUAP). Injury Stages. 2017. 1.

5. Garcia Fernadez FP, Soldevilla Agreda JJ, Pancorbo Hidalgo PL, Verdú Soriano J, López Casanova P, 
Rodríguez Palma M. Documento técnico gneaupp no II. Clasificación-categorización de las lesiones relacionadas con la dependencia. 2 Edición. GNEA UPP; 2014.

6. Restrepo Medrano JC, Rojas JG, Granada Gómez MA. Uso de ácidos grasos en la prevención de úlceras por presión y de extremidad inferior. Av Enferm. 2015; 33(1):133-141.

7. Brunet Rogenski NM, Kurcgant P. Incidencia de las úlceras por presión tras la implementación de un protocolo de prevención. Rev Lat Am Enfermagem. 2012; 20:1-8.

8. Hoyos Porto S, García Arias L, Chavez Carvajal D, Heredia RA. Úlceras por presión en pacientes hospitalizados. Univ Méd. 2015; 56(3):341-355.

9. Redondo JC, Martínez Cuervo F. Prevalencia de úlceras por presión en una zona básica de salud. Gerokomos 2009; 20(2):92-97.

10. Zhao G, Hiltabidel E, Liu Y, Chen L, Liao Y. A cross-sectional descriptive study of pressure ulcer prevalence in a teaching hospital in China. Ostomy Wound Manage. 2010; 56(2):38-42.

11. Kelleher AD, Moorer A, Makic MF. Peer to peer nursing rounds and hospital acquired pressure ulcer prevalence in a surgical intensive care unit: a quality improvement project. J wound, ostomy, Cont Nurs Off Publ Wound, Ostomy Cont Nurses Soc. 2012; 39(2):152-157.

12. González Consuegra RV, Cardona Mazo DM, Murcia Trujillo AP, Matiz Vera GD. Prevalencia de úlceras por presión en Colombia: informe preliminar. Rev la Fac Med. 2014; 62(3):369-377.

13. Torra Bou JE, Rueda López J, Soldevill Agreda JJ, Martinez Cuervo F, Verdú Soriano J. Epidemiología de las úlceras por presión en España en 2013; 4to estudio nacional de prevalencia Gerokomos. 2014; 25(4):162-170.

14. García Fernández FP, Pancorbo Hidalgo PL, Soldevilla Agreda JJ. Predictive capacity of risk assessment scales and clinical judgment for pressure ulcers: a meta-analysis. Journal of Wound Ostomy \& Continence Nursing. 2014; 41(1):24-34.

15. Soldevilla Agreda JJ, Verdú Soriano J, García Fernández FP, Pérez Acevedo G, Sarabia Lavin R, et al. El impacto económico de las lesiones por presión. Revisión integrativa. Gerokomos. 2017; 28(2):8397.

16. Ministerio de la Protección Social. Anexo Técnico. Resolución Número 1446 del 8 mayo de 2006. Por la cual se define el Sistema de Información para la Calidad y se adoptan los indicadores de monitoría del Sistema Obligatorio de Garantía de Calidad de la Atención en Salud. El ministerio. 2006.

17. Ministerio de Sanidad y Política Social. Informe Plan de calidad para el Sistema Nacional de Salud 2006-2010, balance de actividades y acciones previstas. Minist Sanid y política Soc Gob España [Internet]. 2009 [consultado el 20 abril de 2018]; 1-68. Disponible en: http://www.msssi.gob.es/ organizacion/sns/planCalidadSNS/docs/InformePlanCalidad_ESP.pdf

18. Sarabia Lavin R, Soldevilla ágreda JJ. Las úlceras por presión como problema de seguridad del paciente. Gerokomos 2016; 27(4):161-167.

19. Nettel F, Rodríguez N, Nigro J, González M, Conde A, Muñoa A, et al. Primer consenso latinoamericano de úlceras venosas. Rev Mex Angiol [Internet]. 2013 [Consultado el 20 abril de 2018]; 41(1):95-126. Disponible en: http://www.medigraphic.com/pdfs/revmexang/an-2013/an133b.pdf

20. Ministerio de salud. Prevención Úlceras por Presión - paquetes instruccionales- guía técnica "buenas prácticas para la seguridad del paciente en la atención en salud". [Internet]. 2016 [Consultado el 20 abril de 2018]; 1-100. Disponible en: https://www.minsalud.gov.co/sites/rid/Lists/BibliotecaDigital/ RIDE/DE/CA/prevenir-ulceras-por-presion.pdf

21. González García MR. Prevalencia de úlceras por presión en pacientes ingresados en instituciones de salud y residencias geriátricas de Uruguay. [Internet]. 2013 [Consultado el 12 abril de 2018]; 20-38. Disponible en: http://bucserver01.unican.es/xmlui/handle/10902/2603

22. Do Prado Firmino MR, Araújo LO, Sanches Bermudes JP, Mantovani MF, Verdú Soriano J, Castanho 
ISSN-PRINT

1794-9831

E-ISSN 2322-7028

Vol. 15 No. 2

Jul - Dic 2018

Cúcuta, Colombia
Moreira R. Prevalencia de las úlceras de pierna en la región sur de Brasil. Gerokomos 2013; 24(4):179183.

23. González Arjona J, Rodríguez González D, Reyes Marin L, Tapias Gamboa S. Prevalencia de las úlceras por presión y la aplicación de la escala de Braden en los pacientes hospitalizados en el servicio de medicina interna de un hospital de tercer nivel en la ciudad de Cartagena durante en el año 2011. [Trabajo de grado]. Cartagena: Corporación Universitaria Rafael Núñez; 2011.

24. Bautista Montaña IE, Bocanegra Vargas LA. Prevalencia y factores asociados de las úlceras por presión en pacientes hospitalizados en los servicios de medicina interna, neurología y cuidados intensivos de una institución prestadora de salud de III nivel de la ciudad Bogotá en el año 2009. [Trabajo de grado]. Bogotá: Pontificia Universidad Javeriana; 2009.

25. Valero Cárdenas H, Parra DI, Rey Gómez R, Camargo Figuera FA. Prevalencia de úlceras por presión en una institución de salud. Rev Univ Ind Santander Salud. 2011; 43(3):249-255

26. González Consuegra RV, López Zuluaga WJ, Roa Lizcano KT. Epidemiología de lesiones de piel relacionadas con el cuidado: estudio de la prevalencia en Colombia. Rev. Enf. Ref. 2017; serIV(15): $65-72$

27. Flores Montes I, De la Cruz Ortiz S, Ortega Vargas MC, Hernández Morales S, Cabrera Ponce MF. Prevalencia de úlceras por presión en pacientes atendidos en el Instituto Nacional de Cardiología Ignacio Chávez. Rev Mex Enfermería Cardiol. 2010; 18(1-2):13-17.

28. Allman RM. Pressure Ulcers Among the Elderly. N Engl J Med. 1983; 308(2):97-100.

29. Saunders LL, Krause JS, Peters BA, Reed KS. The Relationship of Pressure Ulcers, Race, and Socioeconomic Conditions after Spinal Cord Injury. J Spinal Cord Med. 2010; 33(4):387-395.

30. Soldevilla Ágreda J, Pancorbo Hidalgo PL, Verdú Soriano J, López Casanova P, Rodríguez Palma M. Documento Técnico GNEAUPP No I: Prevención de las Úlceras por Presión. Grupo Nacional para el estudio y asesoramiento en úlceras por presión y heridas crónicas. 2014.

31. Carbonell Fornés P, Murillo Llorente M. Las úlceras por presión en gerontología: prevalencia y variables definitorias de las lesiones y pacientes. Gerokomos. 2015; 26(2):63-67.

32. Lima Serrano M, González Méndez MI, Martín Castaño, Alonso Araujo I, Lima Rodríguez JS. Validez predictiva y fiabilidad de la escala de Braden para valoración del riesgo de úlceras por presión en una unidad de cuidados intensivos Medicina Intensiva 2018; 42(2):82-91. 